\title{
Incentives and Resource Sharing in Spectrum Commons
}

\author{
Junjik Bae*, Eyal Beigman ${ }^{\dagger}$, Randall Berry*, Michael L. Honig*, and Rakesh Vohra ${ }^{\dagger}$ \\ *EECS Department \\ Northwestern University, Evanston, IL 60202 \\ junjik@u.northwestern.edu, \{rberry, mh\}@eecs.northwestern.edu \\ $\dagger$ CMS-EMS, Kellogg School of Management \\ Northwestern University, Evanston, IL 60208 \\ \{e-beigman,r-vohra\}@northwestern.edu
}

\begin{abstract}
It has been suggested that light regulation in the form of etiquette protocols, device design and bargaining amongst users will suffice to mitigate a tragedy of the commons in unlicensed spectrum. In this paper we propose a game theoretic model to examine this claim. In this game, each user decides whether or not to set up an access point, which operates on a particular (single) band. The effect of regulation is modeled in reduced form through transfers. A user who sets up an access point, provides payments to each neighbor who does not and suffers a disutility depending on the number of interfering access points. A user who does not set up an access point, receives payments from each neighbor that does. For a suitable model of payoffs, the game is a potential game and best response updates converge to a Nash equilibrium of the game. For any interference parameters, there is a suitable transfer resulting in a Nash equilibrium which is efficient. However, all Nash equilibria may not be efficient.
\end{abstract}

Index Terms-commons model, tragedy of commons, interference mitigation, equilibrium, potential game

\section{INTRODUCTION}

Due in part to the success of IEEE 802.11 Wi-Fi networks, the "commons model" has been proposed as a paradigm for the efficient use of wireless spectrum [1]-[4]. Anyone may use spectrum, without a license, provided they obey prescribed "spectrum etiquette," such as transmitting with a given peak power constraint or following a common MAC protocol.

Here we consider a commons model in which users may install access points (APs) to serve their own as well as other users' traffic. ${ }^{1}$ In the latter case, the AP owner may receive a payment for this [5]. One concern with such a model is that as the density of APs increases, the users may eventually suffer a "tragedy of the commons," because of increasing interference [6]. It has been suggested that it is enough to lightly regulate a commons to mitigate the tragedy. Schemes such as etiquette protocols, restricted design of devices and bargaining amongst users have been proposed [7], [8]. In this paper we model the effect of regulation in reduced form through a shared rate. Namely, an AP owner can provide

\footnotetext{
This research was supported in part by NSF under grant CNS-0519935.

${ }^{1}$ Although this work is motivated by 802.11 systems, here "Access Point" could refer to other types of systems sharing a common band.
}

payments to neighboring users to encourage them not to set up interfering APs. This payment may take the form of providing discounted service and/or providing a share of revenue to potential interferers.

Given such a scheme, we study a game theoretic model to determine potential user behavior. In this game, each user decides whether or not to set up an AP, which operates on a particular (single) band. If a user sets up an AP, she provides payments to each neighbor who does not and suffers a disutility depending on the number of interfering APs. On the other hand, if a user does not set up an AP, she receives payments from each neighbor that does. Clearly, if the payments are large enough, the user may decide not to set up an AP and thereby reduce interference. For a given model of the agents' payoffs, we show that the resulting game is a potential game and that best response updates converge to a Nash equilibrium of the game.

A game is called a potential game if all players in the game change their strategy as if they jointly optimize a common objective function, i.e. a potential function [9]. Potential games have been used to model various network resource allocation problems, including distributed power control [10]-[12], noncooperative routing in wired networks [13], and multihoming of users to the APs in WLANs [14]. ${ }^{2}$

Using the potential function of the AP deployment game, we analyze the Nash equilibrium under various assumptions. First, we assume that users are located on a two dimension lattice and that interference only comes from the nearest APs. This models a situation where either the density of APs or transmission power of each AP is relatively low. In this case, we show that Nash equilibrium exist in both pure and mixed strategies and that at least one Nash equilibrium achieves the socially optimal density of APs with the appropriate payment. We then account for interference from outside of the nearest neighbors. Our results suggest that as the transmission power increases relative to the node density, implementing such a commons approach becomes more difficult and other forms

\footnotetext{
${ }^{2}$ Other related work, in which a game theoretic approach is used to analyze the performance of ad hoc networks (including IEEE 802.11), is presented in [15]-[19].
} 
of spectrum sharing (e.g. a secondary market) may be more appropriate.

\section{THE MODEL}

Consider a two-dimensional $L_{1} \times L_{2}$ lattice. Every lattice point has a selfish agent who decides whether she sets up an AP or not. Namely, agent $l_{i j}$ at lattice point $(i, j)$ chooses a strategy $y_{i j} \in Y_{i j}$, based on interference and the shared data rate from the nearest APs. This shared data rate among nearest neighbors is the regulatory measure we introduce and is discussed later in detail. The strategy space $Y_{i j}$ of agent $l_{i j}$ is $Y_{i j}=\{0,1\}$, where $y_{i j}=1$ if agent $l_{i j}$ decides to set up an AP and $y_{i j}=0$ if she decides not. If both agents $l_{k l}$ and $l_{i j}$ set up their own APs, the inference from $l_{k l}$ to $l_{i j}$ is given by $I_{k l \rightarrow i j}$. On the other hand, if agent $l_{i j}$ decides not to set up the AP, then she shares rate $\gamma_{k l \rightarrow i j}$ from agent $l_{k l}$ 's AP, assuming agent $l_{k l}$ sets up the AP. ${ }^{3}$ This rate sharing can be justified by the fact that agent $l_{i j}$ is more likely to set up an AP if there is no rate sharing resulting in increased interference to agent $l_{k l}$. If the rate degradation from this interference is severe enough, then agent $l_{k l}$ has an incentive to share her rate with agent $l_{i j}$. For tractability we assume that the nodes are placed at lattice points in the plane, and that rate sharing occurs only between nearest neighbors in the lattice. ${ }^{4}$ Therefore, $\gamma_{k l \rightarrow i j}=0$ if $l_{k l}$ is not in the set of agent $l_{i j}$ 's nearest neighbors, $H_{i j}{ }^{5}$

The payoff function of agent $l_{i j}$ depends on her own strategy as well as those of other agents. Here, we restrict attention to the following payoff function. If agent $l_{i j}$ decides to set up her own AP given the other agents' decisions, her payoff becomes

$$
\begin{aligned}
\pi_{i j}^{A}\left(y_{i j}=1, y_{-i j}\right)= & R-C-\sum_{k l \in\left\{L_{1} \times L_{2}\right\}} y_{k l} \cdot I_{k l \rightarrow i j} \\
& -\sum_{k l \in H_{i j}}\left(1-y_{k l}\right) \cdot \gamma_{i j \rightarrow k l},
\end{aligned}
$$

where $R$ is the total rate generated from her own AP and $C$ is the fixed cost for setting up the AP. $y_{-i j}$ denotes the set of strategies of all agents except agent $l_{i j}$. This payoff function can be motivated by viewing $R$ as the total rate an agent can achieve over a coverage area if there are no interferers, and $I_{k l \rightarrow i j}$ as the reduction in coverage caused by each interfering AP. Of course, assuming this linear relation is a simplification, but it provides a tractable model that captures the key interaction among agents. On the other hand, if agent $l_{i j}$ decides not to set up her own AP, then she shares the rate from the APs in her nearest neighborhood $H_{i j}$ and her payoff

\footnotetext{
${ }^{3}$ We assume payoff functions that are linear in rate and so this rate sharing can be equally viewed as a transfer payment.

${ }^{4}$ We can relax this assumption and allow rate sharing between nonneighboring agents. This relaxation, however, increases the total amount of information that an agent should know before she makes a decision, and may not be practical.

${ }^{5}$ Depending on the boundary condition, the set of nearest neighbors, $H_{i j}$ can be different. For a periodic boundary condition in two-dimensional (torus) lattice, considered in Section IV, the set of nearest neighbors of agent $l_{11}$ is given by $H_{11}=\left\{(1,2),\left(1, L_{1}\right),(2,1),\left(L_{2}, 1\right)\right\}$. Without the periodic boundary condition $H_{11}=\{(1,2),(2,1)\}$.
}

becomes

$$
\pi_{i j}^{N}\left(y_{i j}=0, y_{-i j}\right)=\sum_{k l \in H_{i j}} y_{k l} \cdot \gamma_{k l \rightarrow i j} .
$$

Note that we can write the payoff function of agent $l_{i j}$ as the following:

$$
\pi_{i j}\left(y_{i j}, y_{-i j}\right)=y_{i j} \cdot \pi_{i j}^{A}+\left(1-y_{i j}\right) \cdot \pi_{i j}^{N} .
$$

For the preceding payoff functions, we consider a noncooperative game $\Gamma\left(\pi_{11}, \cdots, \pi_{L_{1} L_{2}}\right)$ among agents in the lattice. In this game, given fixed actions for all other agents, a rational agent decides whether or not she sets up an AP as follows. If $\pi_{i j}^{A} \geq \pi_{i j}^{N}$, then agent $l_{i j}$ sets up the AP at her lattice point. Otherwise, she chooses to share the rate from the APs in nearest neighborhood instead of setting up her own AP. We show later that this game $\Gamma$ is a potential game under certain conditions. This allows us to assert the existence of a Nash equilibrium and characterize the efficiency of Nash equilibria as a function of the amount of rate sharing.

No coordination or no regulatory measure among agents can be represented by $\gamma=0$. Without rate sharing, agent $l_{i j}$ 's payoff if she decides not to set up the AP becomes $\pi_{i j}\left(0, y_{-i j}\right)=0$ from (2). Therefore, an agent is encouraged to set up her own AP unless interference from other APs is large enough such that her payoff with the AP is negative. This can lead to a situation where a large number of agents in the lattice set up the APs and experience severe interference. As we see later in Section VI, the payoffs of all agents can become very low, especially when the density of agents is high. This is an example of the so called a "tragedy of the commons" [6].

\section{A. Model Limitations}

Here we briefly discuss the limitations imposed by the simplifications used to contruct the preceding model, which is analyzed in subsequent sections. First, we assume that the wireless nodes are placed in a lattice, whereas in practice the agents are likely to be randomly distributed over the geographic area of interest. The regular spacing of nodes in a lattice implies that the interference externality imposed by each active node on its nearest neighbor is the same. This enables us to characterize properties of the AP deployment game, such as the existence of equilibria along with the associated efficiency, with a single transfer price. ${ }^{6}$ Of course, the transfer payment scheme can be applied to more general configurations of AP nodes, but then it is likely that different transfer prices would be needed to prove similar results. Such an analysis would be significantly more complicated than that presented here.

The second simplifying assumption is that each AP in the lattice uses the same set of frequencies. Namely, if an agent decides to set up an AP, then she transmits over the entire band. Our model therefore does not directly account for the

\footnotetext{
${ }^{6}$ Also, in Section V we show that the AP deployment game on a lattice is a potential game with different transfer prices across the network.
} 
possibility of using dynamic channel assignment schemes to avoid interference, such as those proposed for IEEE 802.11 in [20]-[23]. (An alternative interpretation of our model is that the particular band considered has already been assigned to each AP by such a channel assignment algorithm, and that the transfer payment scheme is subsequently being used to mitigate interference within that band.)

Finally, as discussed earlier, the payoff depends linearly on the interference. A more accurate model might account for the degradation due to interference by computing the received Signal-to-Interference Plus Noise Ratio at each node. The linear payoff assumed here facilitates tractability while providing insight into the benefits of using transfer payments for more realistic scenarios. We also point out that although we initially consider rate sharing between nearest neighbors in the lattice, we relax this assumption in Section III and show that the AP deployment game is a potential game if rates are shared between non-neighboring agents. However, that increases the total amount of information that an agent needs to make a decision.

\section{Potential Games}

We begin by giving some background on potential games. There are class of games with several desirable properties which we will exploit. First, pure Nash equilibrium strategies exist (assuming finite strategy sets) and are relatively easy to compute using a potential function. Second, in these games, Nash equilibrium can be justified as being the outcome of a boundedly rational learning process such as best response updates.

Let $\Gamma\left(\pi_{1}, \pi_{2}, \ldots, \pi_{n}\right)$ be a game with a finite number of players. The set of players is $N=\{1,2, \ldots, n\}$, the set of strategies of player $i$ is $Y_{i}$, and the payoff function of player $i$ is $\pi_{i}: Y \rightarrow R$, where $Y=Y_{1} \times Y_{2} \times \cdots \times Y_{n}$ is the set of strategy profiles. A function $P: Y \rightarrow R$ is a potential function for $\Gamma$, if for every $i \in N$ and for every $y_{-i} \in Y_{-i}$

$$
\pi_{i}\left(x, y_{-i}\right)-\pi_{i}\left(z, y_{-i}\right)=P\left(x, y_{-i}\right)-P\left(z, y_{-i}\right)
$$

for every $x, z \in Y_{i}$, where $Y_{-i}$ is the Cartesian product of the strategy space of all players except player $i$.

Definition 1: A game $\Gamma$ is called a potential game if it admits a potential function.

Namely, a game is considered a potential game if the improvement of the player's payoff by changing her strategy can be expressed in terms of the potential function which is the same for all players. This definition leads to the following Lemma.

Lemma 1 ([9]): Let $P$ be a potential function for $\Gamma\left(\pi_{1}, \pi_{2}, \ldots, \pi_{n}\right)$. Then the equilibrium set of $\Gamma\left(\pi_{1}, \pi_{2}, \ldots, \pi_{n}\right)$ coincides with the equilibrium set of $\Gamma(P, P, \ldots, P)$. That is, $y \in Y$ is an equilibrium point for $\Gamma$ if and only if for every $i \in N$

$$
P(y) \geq P\left(x, y_{-i}\right) \text { for every } x \in Y_{i} .
$$

Corollary 2 ( [9]): Every finite potential game possesses a pure-strategy equilibrium.
Lemma 1 and Corollary 2 show the existence of a Nash equilibrium and how to compute it using the potential function.

In the potential game with a finite set of strategies, Nash equilibrium can be reached by best response updates [9]. Best response updates of the game $\Gamma\left(\pi_{11}, \cdots, \pi_{L_{1} L_{2}}\right)$ are described by the following. At time $t+1$, an agent $l_{i j}$ is randomly selected among all agents in the lattice $L_{1} \times L_{2}$ and she chooses her strategy, which maximizes her payoff for given strategies of the other agents at time $t$. Namely, agent $l_{i j}$ chooses the best response $y_{i j}(t+1)$ according to

$$
y_{i j}(t+1)=\arg \max _{y_{i j} \in Y_{i j}} \pi_{i j}\left(y_{i j}, y_{-i j}(t)\right)
$$

Initial strategies $y_{i j}(0)$ for all agents are randomly chosen. This best response at time $t+1$ is repeated for $t=0,1,2, \ldots$. with a randomly selected agent. Note that agent $l_{i j}$ 's best response $y_{i j}(t)$ at time $t$ might be different from her best response $y_{i j}\left(t^{\prime}\right)$ at time $t^{\prime} \neq t$. For the AP deployment game, changing actions in this way is reasonable when the fixed cost $C$ is small enough.

Lemma 3: In every finite potential game, a Nash equilibrium can be reached by best response updates.

The following Lemma is useful when we discuss the mixed Nash equilibrium in the potential game considered later.

Lemma 4 ( [9]): Let $\Gamma$ be a finite game. Then $\Gamma$ is a potential game if and only if the mixed extension of $\Gamma$ is a potential game.

\section{Periodic Boundary Condition with Nearest NEIGHBOR INTERFERENCE}

Initially we study the game discussed in Section II, assuming a periodic boundary condition for the lattice $L_{1} \times$ $L_{2}{ }^{7}$ Namely, $L_{1}+1=1$ and $L_{2}+1=1$; Then, the set of the nearest neighbors of $(1,1)$, for example, is $H_{11}=\left\{(1,2),\left(1, L_{2}\right),(2,1),\left(L_{1}, 1\right)\right\}$. This assumption simplifies our analysis by removing boundary effects. (In Section V we consider a game without this periodic boundary condition.) Furthermore, we assume that interference comes only from the nearest neighbor APs. This nearest neighbor interference models a situation where either the density of APs or transmission power of each AP is relatively low. Therefore,

$$
I_{k l \rightarrow i j}= \begin{cases}I, & (k, l) \in H_{i j}, \\ 0, & (k, l) \notin H_{i j},\end{cases}
$$

where $I$ is a constant which represents the interference level between two neighboring APs. The payoff function (3) of

\footnotetext{
${ }^{7}$ The resulting lattice is also called a torus-lattice.
} 
agent $l_{i j}$, then, is given by

$$
\begin{aligned}
& \pi_{i j}\left(y_{i j}, y_{-i j}\right) \\
& =y_{i j} \cdot\left(R-C-\sum_{k l \in H_{i j}} y_{k l} \cdot I-\sum_{k l \in H_{i j}}\left(1-y_{k l}\right) \cdot \gamma\right) \\
& \quad+\left(1-y_{i j}\right) \cdot\left(\sum_{k l \in H_{i j}} y_{k l} \cdot \gamma\right) \\
& =y_{i j} \cdot\left(R-C-\sum_{k l \in H_{i j}} y_{k l} \cdot I-\sum_{k l \in H_{i j}} \gamma\right) \\
& \quad+\sum_{k l \in H_{i j}} y_{k l} \cdot \gamma .
\end{aligned}
$$

For the time being, we assume that the shared rate from the AP of each agent $l_{k l} \in H_{i j}$ to each agent $l_{i j}$ is $\gamma_{k l \rightarrow i j}=\gamma$. Namely, it is the same for all agents in the lattice.

Let $\Gamma_{P B N N}$ denote the resulting game under these assumptions.

\section{Lemma 5:}

$$
\begin{aligned}
& P\left(y_{11}, y_{12}, \ldots, y_{L_{1} L_{2}}\right) \\
& =(R-C)\left(\sum_{i=1}^{L_{1}} \sum_{j=1}^{L_{2}} y_{i j}\right)-\sum_{i=1}^{L_{1}} \sum_{j=1}^{L_{2}} y_{i j}\left(\sum_{k l \in H_{i j}} \gamma\right) \\
& \quad-\frac{1}{2} \sum_{i=1}^{L_{1}} \sum_{j=1}^{L_{2}} y_{i j}\left(\sum_{k l \in H_{i j}} y_{k l} \cdot I\right) .
\end{aligned}
$$

is a potential function for $\Gamma_{P B N N}$.

The proof of this follows by noting that for given $y_{-i j}$,

$$
\begin{aligned}
\pi_{i j}\left(y_{i j}^{\prime}, y_{-i j}\right) & -\pi_{i j}\left(y_{i j}, y_{-i j}\right) \\
& =P\left(y_{i j}^{\prime}, y_{-i j}\right)-P\left(y_{i j}, y_{-i j}\right),
\end{aligned}
$$

for all $y_{i j}, y_{i j}^{\prime} \in Y_{i j}$ and all agents in the Lattice $L_{1} \times L_{2}$. Therefore, according to Definition $1, \Gamma_{P B N N}$ is a potential game with the potential function $P$ given by (9).

On the other hand, the social welfare is the sum of the payoffs of all agents and is not affected by the shared rate $\gamma$ since it is an exchange between two agents. The social welfare of all agents in the $L_{1} \times L_{2}$ lattice is, therefore, given by

$$
\begin{aligned}
& S W\left(y_{11}, y_{12}, \ldots, y_{L_{1} L_{2}}\right) \\
& =(R-C)\left(\sum_{i=1}^{L_{1}} \sum_{j=1}^{L_{2}} y_{i j}\right)-\sum_{i=1}^{L_{1}} \sum_{j=1}^{L_{2}} y_{i j}\left(\sum_{k l \in H_{i j}} y_{k l} \cdot I\right) .
\end{aligned}
$$

We next compare a Nash equilibrium strategy with a strategy which maximizes the social welfare and discuss the efficiency loss at the Nash equilibrium.

\section{A. Pure Nash Equilibrium}

The first-order derivative of the potential function (9) is given by

$$
\frac{\partial P}{\partial y_{i j}}=(R-C)-\sum_{k l \in H_{i j}} \gamma-\sum_{k l \in H_{i j}} y_{k l} \cdot I .
$$

Note that $\frac{\partial P}{\partial y_{i j}}$ does not depend on $y_{i j}$. If the strategy space is restricted to $\{0,1\}$ for all agents, the best response of agent $l_{i j}$ for $\frac{\partial P}{\partial y_{i j}}>0\left(\frac{\partial P}{\partial y_{i j}}<0\right)$ is $y_{i j}=1\left(y_{i j}=0\right)$. By Lemma 3, best response updates of randomly selected agents converge to a Nash equilibrium. In a similar way, if $\frac{\partial S W}{\partial y_{i j}}>0\left(\frac{\partial S W}{\partial y_{i j}}<0\right)$, then $y_{i j}=1\left(y_{i j}=0\right)$ to maximize the social welfare given by (11).

Let $H_{i j}^{A}$ be the set of nearest neighbors of agent $l_{i j}$ which set up an AP. Then from (12), it can be seen that agent $l_{i j}$ 's action in a Nash equilibrium is to set up an AP if

$$
\frac{R-C-\sum_{k l \in H_{i j}} \gamma}{I}>\left|H_{i j}^{A}\right|
$$

and not to set up an AP when

$$
\frac{R-C-\sum_{k l \in H_{i j}} \gamma}{I}<\left|H_{i j}^{A}\right| .
$$

The agent is indifferent when equality holds. For $\Gamma_{P B N N}$, $\sum_{k l \in H_{i j}} \gamma=4 \gamma$ for all $l_{i j}$, and so the above threshold on the number of neighbors is

$$
\mathbf{H}_{t h}=\frac{R-C-4 \gamma}{I} .
$$

There are 5 cases of interest for this quantity.

1) $0<\mathbf{H}_{t h}<1$ : In this case, an agent will set up an AP in a Nash equilibrium only if none of her neighbors does. For fixed value of $R-C$, this is true if $\gamma$ and $I$ satisfy

$$
\left\{\begin{array}{l}
R-C-4 \gamma-I \cdot 0>0 \\
R-C-4 \gamma-I \cdot 1<0
\end{array}\right.
$$

In addition, we assume $\gamma \leq I$. Otherwise, an agent would always prefer having a neighboring AP over sharing the rate. Similarly, the following conditions must hold for an agent to set up an AP to maximize $S W$ only when there is no AP in the nearest neighborhood:

$$
\left\{\begin{array}{l}
R-C-2 I \cdot 0>0 \\
R-C-2 I \cdot 1<0
\end{array}\right.
$$

2) $1<\mathbf{H}_{t h}<2$ : In this case, an agent will set up an AP in a Nash equilibrium only if no more than one of her neighbors does. $\gamma$ and $I$ must satisfy

$$
\left\{\begin{array}{l}
R-C-4 \gamma-I \cdot 1>0 \\
R-C-4 \gamma-I \cdot 2<0
\end{array}\right.
$$

The analogous conditions for the social welfare must hold:

$$
\left\{\begin{array}{l}
R-C-2 I \cdot 1>0 \\
R-C-2 I \cdot 2<0
\end{array}\right.
$$

3) $2<\mathbf{H}_{t h}<3$ : An agent will set up an AP in a Nash equilibrium only if no more than two her neighbors do. For this case, $\gamma$ and $I$ must satisfy

$$
\left\{\begin{array}{l}
R-C-4 \gamma-I \cdot 2>0 \\
R-C-4 \gamma-I \cdot 3<0
\end{array}\right.
$$

and

$$
\left\{\begin{array}{l}
R-C-2 I \cdot 2>0 \\
R-C-2 I \cdot 3<0
\end{array}\right.
$$




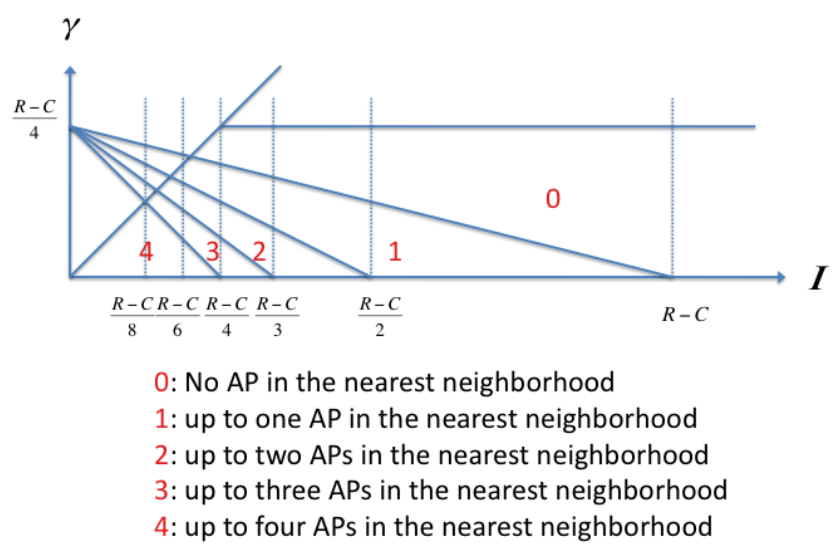

Fig. 1. Interference $I$ and shared rate $\gamma$ for pure Nash equilibrium depending on the maximum number of APs allowed in the nearest neighbors.

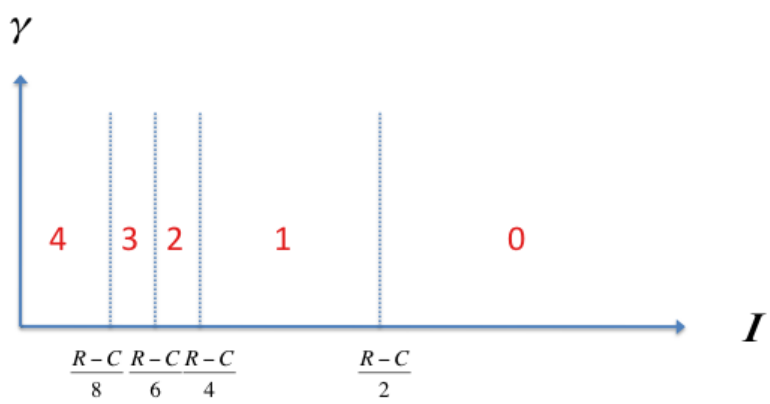

$0:$ No AP in the nearest neighborhood

1: up to one AP in the nearest neighborhood

2: up to two APs in the nearest neighborhood

3: up to three APs in the nearest neighborhood

4: up to four APs in the nearest neighborhood

Fig. 2. Interference $I$ and shared rate $\gamma$ for the optimal social welfare depending on the maximum number of APs allowed in the nearest neighbors.

gives the same condition for the social welfare.

For the remaining two cases $\left(3<\mathbf{H}_{t h}<4\right.$ and $\left.\mathbf{H}_{t h}>4\right)$, similar conditions for the potential function $P$ and the social welfare $S W$ can be obtained easily and we omit them here.

Figure 1 and Figure 2 summarize these cases. Figure 1 shows the values of $I$ and $\gamma$ for pure strategy Nash equilibria to exist in each case. These values lie in one of five regions; each region is labeled with the maximum number of neighboring APs for which an agent's best response will be to set up an AP (e.g., region 0 corresponds to case $0<\mathbf{H}_{t h}<1$ ). Figure 2 shows the values of $I$ and $\gamma$ for the socially optimum solution to have the same structure.

Proposition 6: Consider the game $\Gamma_{P B N N}$ with the set of strategies $Y_{i j}=\{0,1\}$. For given interference level $I$, there exist a set of $\gamma$ such that a Nash equilibrium achieves the optimal social welfare.

For given interference level $I$, there exist a set of $\gamma$ such that a Nash equilibrium achieves the optimal social welfare. For example, if $I \geq \frac{R-C}{2}$, a set of $\gamma$ which satisfies the conditions of the potential function in case $0<\mathbf{H}_{t h}<1$ induces a Nash equilibrium which might correspond to the optimal strategy. On the other hand, if $I \in\left[\frac{R-C}{4}, \frac{R-C}{2}\right]$, a set of $\gamma$ which satisfies the conditions of the potential function in case $2<$ $\mathbf{H}_{t h}<3$ might generate a Nash equilibrium in which there exists an AP with two APs in the nearest neighborhood. This Nash equilibrium can not be the socially optimal strategy.

Nash equilibrium may not be unique in the game. Let us consider $3 \times 3$ lattice with the periodic boundary condition as an example. If $\gamma$ and $I$ are such that no AP in the nearest neighborhood $H_{i j}$ is allowed when agent $l_{i j}$ sets up an AP $\left(0<\mathbf{H}_{t h}<1\right)$, there exists only one Nash equilibrium with three APs in the lattice. Namely, three among 9 agents decide to set up the APs at Nash equilibrium. On the other hand, if up to two APs are allowed in the nearest neighborhood $\left(2<\mathbf{H}_{t h}<3\right)$, there exist two Nash equilibria with either 5 or $6 \mathrm{APs}$ in the lattice.

\section{B. Mixed Nash Equilibrium}

We next consider the mixed extension of the potential game $\Gamma_{P B N N}$. In this game, each agent $l_{i j}$ can be viewed as having strategy space $Y_{i j}=[0,1]$, where an action $y_{i j} \in Y_{i j}$ can be viewed as the probability that $l_{i j}$ sets up an AP. An agent's payoff is then the expected value of (3) with respect to the actions chosen by every other agent. Since the payoff is linear in the action, it can be seen that the first-order derivative of the potential function is given by (12) and the second-order derivative is given by

$$
\frac{\partial^{2} P}{\partial y_{i j} \partial y_{k l}}= \begin{cases}-I, & (k, l) \in H_{i j} \\ 0, & (k, l) \notin H_{i j} .\end{cases}
$$

Since the Hessian of the potential function $P$ is negative semidefinite, $P$ is a concave function with a unique global maximum. In addition, $\frac{\partial P}{\partial y_{i j}}=\frac{\partial \pi_{i j}}{\partial y_{i j}}$ for all agents $l_{i j} \in L_{1} \times L_{2}$. Therefore, best response updates of agents in the potential game reach this global maximum of the potential function, which corresponds to the unique mixed Nash equilibrium. This gives the following proposition.

Proposition 7: $\Gamma_{P B N N}$ has a unique mixed strategy Nash equilibrium, which is symmetric.

The mixed extension of $\Gamma_{P B N N}$ can be interpreted as a game in which all agents install an AP, but only use it a fraction of the time, indicated by $y_{i j}$. Individual agents do not coordinate their usage.

Assume each agent chooses the same strategy, namely, $y_{11}=y_{12}=\cdots=y_{L_{1} L_{2}}=y$. We can then rewrite the potential function as

$$
P(y)=\left(L_{1} \cdot L_{2}\right)\left\{(R-C-4 \gamma) y-2 I y^{2}\right\},
$$

and so

$$
\frac{\partial P(y)}{\partial y}=\left(L_{1} \cdot L_{2}\right)\{(R-C-4 \gamma)-4 I y\} .
$$




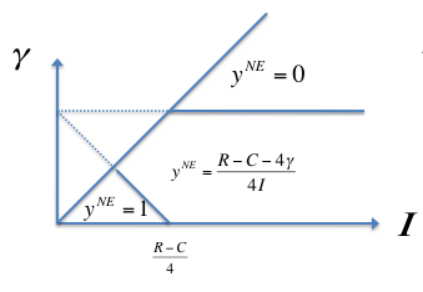

(a) Mixed Nash Equilibrium

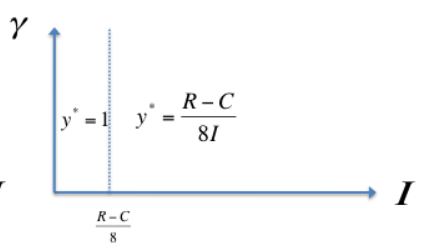

(b) Optimal Social Welfare
Fig. 3. Interference $I$ and shared rate $\gamma$ for mixed Nash equilibrium and social optimum.

It follows that the mixed Nash equilibrium strategies are given by $y_{i j}^{\mathrm{NE}}=y^{\mathrm{NE}}$ for all agents, where

$$
y^{\mathrm{NE}}= \begin{cases}0, & \frac{R-C-4 \gamma}{4 I}<0 \\ 1, & \frac{R-C-4 \gamma}{4 I}>1, \\ \frac{R-C-4 \gamma}{4 I}, & \text { otherwise. }\end{cases}
$$

Similarly, assuming symmetric strategies, the social welfare $^{8}$ and its first-order derivative are given by

$$
S W(y)=\left(L_{1} \cdot L_{2}\right)\left\{(R-C) y-4 I y^{2}\right\},
$$

and

$$
\frac{\partial S W(y)}{\partial y}=\left(L_{1} \cdot L_{2}\right)\{(R-C)-8 I y\} .
$$

It follows that the social welfare is maximized if $y_{i j}=y^{*}$ for all $l_{i j}$, where

$$
y^{*}= \begin{cases}0, & \frac{R-C}{8 I}<0 \\ 1, & \frac{R-C}{8 I}>1 \\ \frac{R-C}{8 I}, & \text { otherwise }\end{cases}
$$

Figure 3 shows the values of $I$ and $\gamma$ corresponding to different mixed Nash equilibria and the optimal social welfare. The overlapped region where $y^{\mathrm{NE}}=1$ and $y^{*}=1$ is such that the mixed Nash equilibrium achieves the optimal social welfare. In general, however, the social welfare at Nash equilibrium does not correspond to the optimal social welfare. We consider the efficiency of the Nash equilibrium for three possible range of $I$ next.

1) $I \in\left[0, \frac{R-C}{8}\right]:$ As we can see from Figure 3 , for given interference $I \in\left[0, \frac{R-C}{8}\right]$, the mixed strategy Nash equilibrium is $y^{\mathrm{NE}}=1$ and the probability for setting up an AP to achieve the optimal social welfare is $y^{*}=1$. Therefore, the efficiency at the Nash equilibrium, defined by the ratio of the social welfare at $y^{\mathrm{NE}}$ vs. at $y^{*}$ is given by

$$
\varepsilon=\frac{S W\left(y^{\mathrm{NE}}=1\right)}{S W\left(y^{*}=1\right)}=1,
$$

regardless of $\gamma \in[0, I]$.

\footnotetext{
${ }^{8}$ The social welfare function is also a concave function since its Hessian is given by

$$
\frac{\partial^{2} S W}{\partial y_{i j} \partial y_{k l}}= \begin{cases}-2 I, & (k, l) \in H_{i j} \\ 0, & (k, l) \notin H_{i j}\end{cases}
$$
}

and is a negative semi-definite.
2) $I \in\left[\frac{R-C}{8}, \frac{R-C}{4}\right]:$ In this case, there are two possible regions depending on the shared rate $\gamma$. If $0 \leq \gamma \leq \frac{R-C-4 I}{4}$, the Nash equilibrium strategy is $y^{\mathrm{NE}}=1$, while the optimal probability is $y^{*}=\frac{R-C}{8 I}$. The efficiency is then given by

$$
\varepsilon=\frac{S W\left(y^{\mathrm{NE}}=1\right)}{S W\left(y^{*}=\frac{R-C}{8 I}\right)}=\frac{16 I}{(R-C)}-\frac{64 I^{2}}{(R-C)^{2}},
$$

and, therefore, the efficiency does not depend on the shared rate $\gamma$. On the other hand, if $\frac{R-C-4 I}{4} \leq \gamma \leq I$, the Nash equilibrium strategy and the optimal probability are $y^{\mathrm{NE}}=$ $\frac{R-C-4 \gamma}{4 I}$ and $y^{*}=\frac{R-C}{8 I}$ respectively. The efficiency is

$$
\begin{aligned}
\varepsilon & =\frac{S W\left(y^{\mathrm{NE}}=\frac{R-C-4 \gamma}{4 I}\right)}{S W\left(y^{*}=\frac{R-C}{8 I}\right)} \\
& =4\left\{\frac{R-C-4 \gamma}{R-C}-\left(\frac{R-C-4 \gamma}{R-C}\right)^{2}\right\} .
\end{aligned}
$$

Since $\frac{R-C-4 I}{4} \leq \gamma \leq I$, the range of the efficiency for given $I$ is $\varepsilon_{\min } \leq \varepsilon \leq 1$, where $\varepsilon_{\min }$ is given by (30).

3) $I \in\left[\frac{R-\bar{C}}{4}, \infty\right]:$ Similarly, there are two possible regions depending on the shared rate $\gamma$. If $0 \leq \gamma \leq \frac{R-C}{4}$, the efficiency is given by (31) and the range is $0 \leq \varepsilon \leq 1$. On the other hand, if $\frac{R-C}{4} \leq \gamma \leq I$, the efficiency is $\varepsilon=0$ because $y^{\mathrm{NE}}=0$.

Proposition 8: For any $R, C$, and $I$, there exist a $\gamma$ so that the unique mixed strategy Nash equilibrium of $\Gamma_{P B N N}$ is efficient.

If the interference level is $I \in\left[0, \frac{R-C}{8}\right]$, the mixed strategy Nash equilibrium is efficient for any $\gamma \leq I$. If $I>\frac{R-C}{8}$, the rate sharing should be $\gamma=\frac{R-C}{8}$ to have an efficient Nash equilibrium.

\section{Generalization}

In this section, we generalize the previous results. First, we relax the constraint on the same shared rate $\gamma$ among all agents in the lattice and allow this rate to be different. The shared rate from the AP of agent $l_{k l}$ to agent $l_{i j}$ is denoted by $\gamma_{k l \rightarrow i j}$. Assuming $\gamma_{k l \rightarrow i j}=\gamma_{i j \rightarrow k l},{ }^{9}$ we can show that the potential function is now given by

$$
\begin{aligned}
& P\left(y_{11}, y_{12}, \ldots, y_{L_{1} L_{2}}\right) \\
& =(R-C)\left(\sum_{i=1}^{L_{1}} \sum_{j=1}^{L_{2}} y_{i j}\right)-\sum_{i=1}^{L_{1}} \sum_{j=1}^{L_{2}} y_{i j}\left(\sum_{k l \in H_{i j}} \gamma_{k l \rightarrow i j}\right) \\
& \quad-\frac{1}{2} \sum_{i=1}^{L_{1}} \sum_{j=1}^{L_{2}} y_{i j}\left(\sum_{k l \in H_{i j}} y_{k l} \cdot I\right) .
\end{aligned}
$$

Second, we can remove the periodic boundary condition and consider edge effects in the lattice. We can still show that the game is a potential game with the potential function in (32). Note that $H_{i j}$ does not always contain 4 nearest neighbors as with the periodic boundary condition. In addition, if mixed

\footnotetext{
${ }^{9}$ If this condition does not hold, (32) is not a potential function anymore.
} 
strategy Nash equilibria are considered (as in Section IV-B), the potential function (32) can be simplified to

$$
\begin{aligned}
P & (x, y, z) \\
= & (R-C) L^{2}-4(L-2)^{2} \gamma x-12(L-2) \gamma y-8 \gamma z \\
& -2\left(L^{2}-5 L+6\right) I x^{2}-4(L-2) I x y \\
& -4(L-3) I y^{2}-8 I y z,
\end{aligned}
$$

where $x \in[0,1]$ is the strategy of an agent who has 4 nearest neighbors, $y \in[0,1]$ is that of an agent who has 3 nearest neighbors, and $z \in[0,1]$ is that of an agent who has 2 nearest neighbors. Here, we assume $L_{1}=L_{2}=L$ for simplicity. Since the potential function (33) is a concave function, the mixed Nash equilibrium can again be found easily.

Finally, we can include interference from APs beyond the nearest neighbors. This might be relevant when the density of APs increases or the transmission power of each AP increases relative to the node density. Assuming interference only depends on the distance between two APs, interference from the AP of agent $l_{k l}$ to the AP of agent $l_{i j}$ is given by

$$
I_{k l \rightarrow i j}=\frac{I}{|(k, l)-(i, j)|^{a}},
$$

where $a$ is the path-loss exponent. Note that $I_{k l \rightarrow i j}=I_{i j \rightarrow k l}$. The potential function of the game is then given by

$$
\begin{aligned}
P & \left(y_{11}, y_{12}, \ldots, y_{L_{1} L_{2}}\right) \\
= & (R-C)\left(\sum_{i=1}^{L_{1}} \sum_{j=1}^{L_{2}} y_{i j}\right)-\sum_{i=1}^{L_{1}} \sum_{j=1}^{L_{2}} y_{i j}\left(\sum_{k l \in H_{i j}} \gamma_{k l \rightarrow i j}\right) \\
& -\frac{1}{2} \sum_{i=1}^{L_{1}} \sum_{j=1}^{L_{2}} y_{i j}\left(\sum_{k l \in\left\{L_{1} \times L_{2}\right\}} y_{k l} \cdot I_{k l \rightarrow i j}\right) .
\end{aligned}
$$

Here, we are still assuming that rate sharing is only between nearest neighbors. As discussed in Section II, we can relax this constraint. The social welfare function is the same as in (11) except that the interference from the APs beyond the nearest neighbors is included. As we see in Section VI, if interference is severe, then an agent is less likely to set up her own AP at a Nash equilibrium even when $\gamma>0$ and this reduces the sum of the agents' payoffs in the lattice. This suggests that as interference becomes severe due to the increase of AP density, implementing such a commons approach becomes more difficult and other forms of spectrum sharing (e.g. a secondary market) may be more appropriate.

\section{Vi. Simulation Results}

We now present simulation results to illustrate properties of the AP deployment game. We begin with a game with periodic boundary conditions and nearest neighbor interference, as in Figure 4. The strategy space of an agent in the game is $Y_{i j}=\{0,1\}$ for all agents $l_{i j}$. Figure 5 shows one realization of the convergence of best response updates to a pure Nash equilibrium with parameters $L_{1}=L_{2}=100, R=10, C=$ $3, I=1.6$ and $\gamma=0.7$. Initial decisions of agents in the

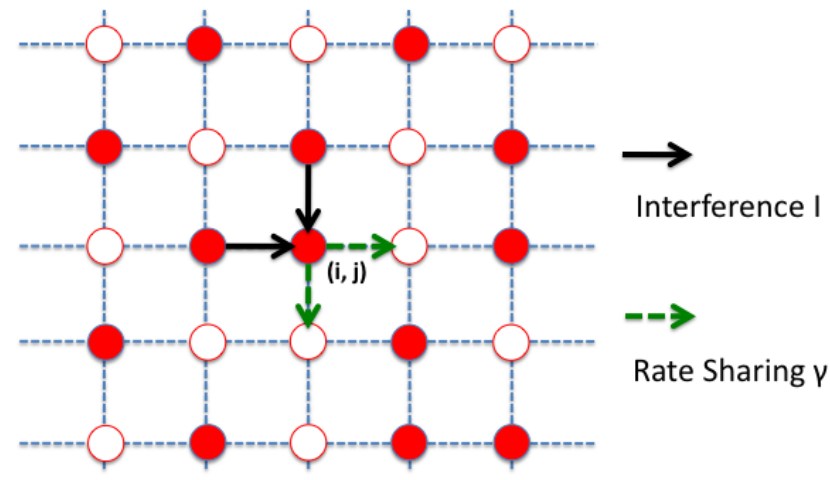

Set up AP

No AP

Fig. 4. Agents in the lattice decide whether or not to set up their own APs. Here we only consider interference from the nearest neighbor APs.
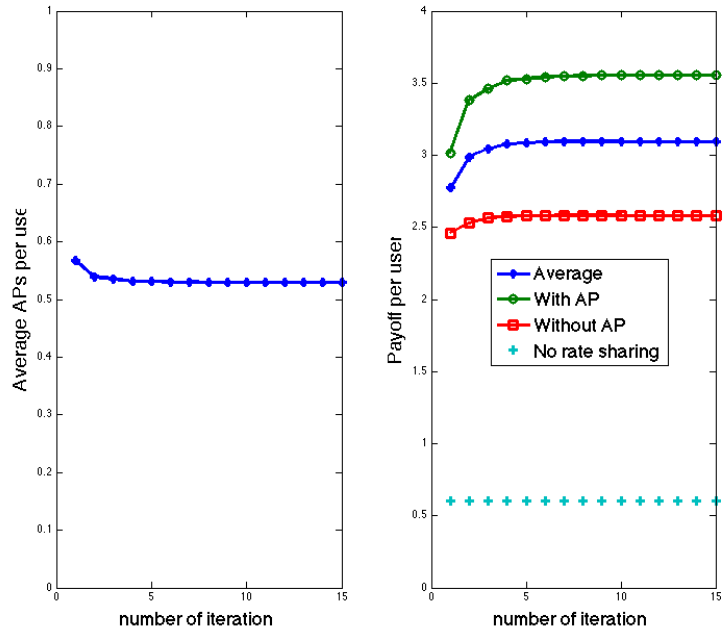

Fig. 5. Game in the lattice with a periodic boundary condition. $L_{1}=L_{2}=$ $100, R=10, C=3, I=1.6$ and $\gamma=0.7$. (a) Average number of APs per agent (b) Average payoff per agent for different decisions.

lattice are randomly chosen. At each iteration, all agents in the lattice are randomly ordered and sequentially choose their best response. Therefore, during one iteration in Figure 5, $100 \times 100=10^{4}$ agents make their decisions.

The parameters considered above allow up to two nearest APs at a pure Nash equilibrium, which corresponds to case $2<\mathbf{H}_{t h}<3$ in Section IV-A3. As we can see easily, the optimal configuration with these parameters is to have chess board like deployment of APs in the lattice and there is no AP in the nearest neighborhood when a user sets up her own AP. Therefore, the average number of APs per agent (per lattice point) at the optimal configuration is 0.5 with the average payoff per agent $\frac{10-3}{2}=3.5$. On the other hand, in the commons model without shared rate $(\gamma=0)$, every 

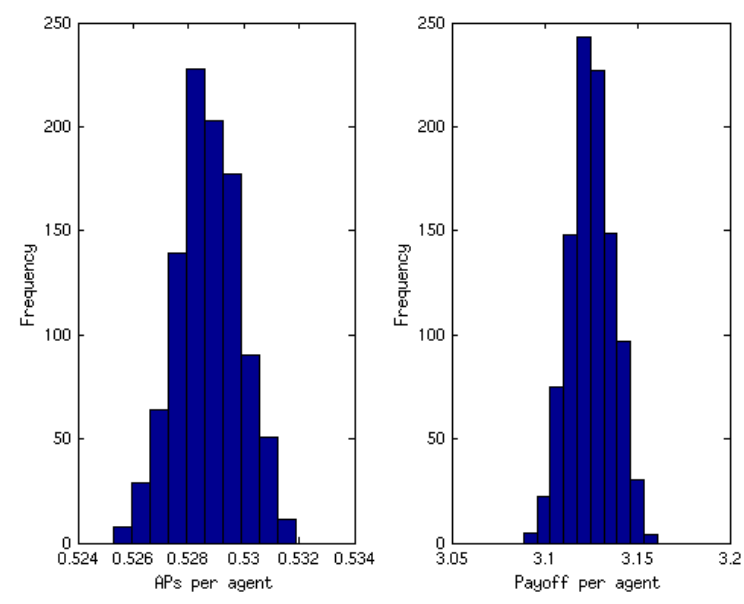

Fig. 6. Histogram of average number of APs and average payoff per agent at Nash equilibrium. Total number of simulation run is 1000. $L_{1}=L_{2}=$ $100, R=10, C=3, I=1.6$ and $\gamma=0.7$.

selfish agent in the lattice sets up her own AP. This reduces the average payoff per agent to $10-3-4 \times 1.6=0.6$, which is substantially lower than the payoff at the optimal configuration. As discussed in Section II, this outcome can be viewed as a "tragedy of commons".

Now we consider the shared rate $\gamma=0.7$. From Section IV, this allows up to two APs in the nearest neighborhood at a Nash equilibrium. Best response updates of agents in the game converge to a Nash equilibrium with more desirable average payoff per agent. After transition period, Figure 5 shows that the average number of APs per agent converges to $\sim 0.527$ and the average payoff per agent to $\sim 3.1$, which are closer to the socially optimal configuration of APs. In addition, it shows the average payoff per agent with and without an AP. We achieve this near optimality simply by introducing the rate sharing among agents.

Note that the Nash equilibrium is not unique as we discussed before and the final results (average number of APs and average payoff per agent) might be different at each simulation run. With large lattice size, however, these differences are relatively small. We simulated 1000 times with the same parameters and our results (See Figure 6) show that the means of the average number of APs and the average payoff per agent over 1000 realizations are 0.5287 (standard deviation 0.0011 ) and 3.1254 (standard deviation 0.0116 ) respectively. However, this does not mean we sampled all possible Nash equilibria in the simulation. In fact, we did not realize the optimal density 0.5 even though it is possible with the parameters we consider.

Figure 7 shows one realization of the average number of APs and the average payoff per agent as a function of the shared rate $\gamma$. If we consider many realizations of the simulation, each point in the figure should be replaced by a distribution such as Figure 6. The overall trend of the figure with mean values, however, will be the same. From the figure, we can see that over a wide range of $\gamma$, the average payoff
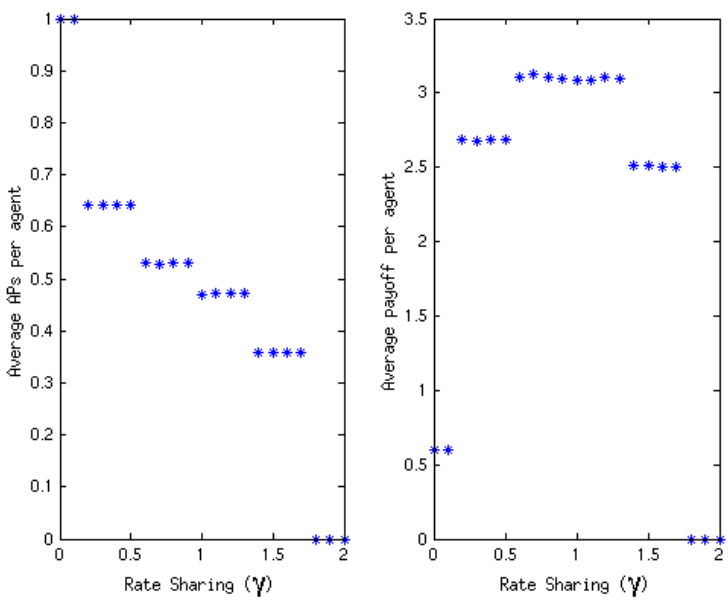

Fig. 7. Average number of APs and average payoff per agent at Nash equilibrium as a function of the shared rate $\gamma$. Every point in the Figure is one particular realization. Other parameters are the same $\left(L_{1}=L_{2}=\right.$ $100, R=10, C=3, I=1.6)$.

per agent is close to the optimal value.

Now we remove the periodic boundary condition from the game. Agents at the edge of the lattice have fewer nearest neighbors and this encourages them to set up the APs. One realization shows that the average APs per agent increases to $\sim 0.546$. Average payoff per agent does not change much. We further generalize this game and include interference from the APs beyond the nearest neighbors. As we expect, less agents decide to set up their own APs due to excessive interference. If the path-loss exponent in (34) is assumed to be $a=4$, the average number of APs per agent is $\sim 0.475$ (average payoff per agent $\sim 2.15$ ). With the path-loss exponent $a=3$, the average number of APs per agent becomes $\sim 0.357$ (average payoff per agent $\sim 1.30$ ).

As interference increases, more agents decide not to set up the AP even there is no APs around with which she can share the rate. This configuration with relatively low density of APs in the lattice is close to the optimal configuration with severe interference. Our calculations in Appendix A show that the optimal density of APs per agent is $\sim 0.424$ when $a=4$ and $\sim 0.330$ when $a=3$. However, even if the shared rate scheme achieves the near optimal configuration, overall average payoff per agent becomes small because of the commons model itself. When interference becomes severe, a frequency division scheme with some frequency reuse factor might increase the average payoff substantially compared to the commons model.

\section{CONCLUSION}

We have considered a game theoretic model of a spectrum commons where non-cooperative users in a lattice decide whether or not to set up their own APs. A simple regulatory measure, the rate sharing, is proposed to mitigate the tragedy of the commons. We have shown that the AP deployment game in the lattice under the periodic boundary condition 
with nearest neighbor interference is the potential game and there exist pure and mixed Nash equilibrium. Moreover, by choosing the shared rate appropriately, we achieve a Nash equilibrium in the game which is efficient. However, with pure strategies other non-efficient equilibria may also exist. The potential game is also generalized to the case where interference comes from the APs beyond the nearest neighbors. The density of APs in the lattice decreases at the Nash equilibrium as interference becomes severe. This result suggests that as interference becomes severe due to the increase of AP density, implementing such a commons approach becomes more difficult and other forms of spectrum sharing (e.g. a secondary market) may be more appropriate.

\section{APPENDIX A}

\section{Optimal Density of APs With Long RANGE INTERFERENCE}

We would like to compute the optimal density of APs when there exists interference from APs beyond the nearest neighbors. This is, however, difficult because of integer optimization nature of the problem. Here, we introduce the mean-field theory in physics [24] and solve this problem approximately. ${ }^{10}$

Since interference from the AP at distance $r$ is proportional to $1 / r^{a}$ as shown in (34), we ignore interference from the APs at distance $r>D_{m}$. In addition, the effective interference radius, denoted by $D_{m}$, is assumed to be $D_{m} \ll L_{1}$ and $D_{m} \ll L_{2}$. The social welfare function is, then, given by

$$
\begin{aligned}
& S W\left(y_{11}, y_{12}, \ldots, y_{L_{1} L_{2}}\right) \\
& =(R-C)\left(\sum_{i=1}^{L_{1}} \sum_{j=1}^{L_{2}} y_{i j}\right) \\
& \quad-\sum_{i=1}^{L_{1}} \sum_{j=1}^{L_{2}} y_{i j}\left(\sum_{|k l-i j| \leq D_{m}} y_{k l} \cdot I_{k l \rightarrow i j}\right) .
\end{aligned}
$$

If we focus on a particular lattice site $(i, j)$, the interactions of this site with others are described by

$$
\begin{aligned}
& S W_{i j}=(R-C) y_{i j}-y_{i j}\left(\sum_{|k l-i j|=D_{1}} y_{k l} \cdot I_{D_{1}}\right. \\
& \left.+\sum_{|k l-i j|=D_{2}} y_{k l} \cdot I_{D_{2}}+\cdots+\sum_{|k l-i j|=D_{m}} y_{k l} \cdot I_{D_{m}}\right),
\end{aligned}
$$

where $D_{n}$ is the distance of $\mathrm{n}$-th nearest neighbors from the site $(i, j)$ and $I_{D_{n}}=\frac{I}{\left|D_{n}\right|^{a}}$ is interference from the n-th nearest neighbor AP. As an approximation we replace the sum over these neighbors in the second term by its mean value, i.e.,

\footnotetext{
${ }^{10}$ We can have the exact solution of the optimal density for one-dimensional lattice.
}

we put

$$
\begin{aligned}
H_{m} & \equiv\left\langle\sum_{|k l-i j|=D_{1}} y_{k l} \cdot I_{D_{1}}+\cdots+\sum_{|k l-i j|=D_{m}} y_{k l} \cdot I_{D_{m}}\right\rangle \\
& =<y>\cdot\left(\sum_{n=1}^{m} N_{D_{n}} \cdot I_{D_{n}}\right),
\end{aligned}
$$

where $\langle\cdot\rangle$ represents an ensemble average and $N_{D_{n}}$ is the number of nearest neighbors at distance $D_{n}$.

$H_{m}$ should be determined in such a way that it leads to a self-consistent solution of the statistical problem. First, we define the energy of the lattice $(i, j)$ by

$$
e_{i j} \equiv-S W_{i j}=-\left(H-H_{m}\right) \cdot y_{i j}
$$

where $H=R-C$. Since $y_{i j} \in\{0,1\}$, the ensemble average of the strategy, or $\langle y\rangle$ is given by

$$
\begin{aligned}
<y>=1 \times \operatorname{Prob}\left(e_{i j}=-\left(H-H_{m}\right)\right) \\
+0 \times \operatorname{Prob}\left(e_{i j}=0\right) \\
=\frac{e^{\beta\left(H-H_{m}\right)}}{1+e^{\beta\left(H-H_{m}\right)}}
\end{aligned}
$$

from statistical physics. From (38) and (40), we can compute $H_{m}$ and, therefore, $\langle y\rangle$. The parameter should be $\beta \rightarrow \infty$ to get $\langle y\rangle_{\text {opt }}$, the average number of APs per user when the optimal social welfare is achieved. Therefore, $\langle y\rangle_{\text {opt }}$ is given by

$$
<y>_{\text {opt }}=\left\{\begin{array}{cl}
1, & \sum_{n=1}^{m} N_{D_{n}} \cdot I_{D_{n}} \leq H, \\
\frac{H}{\sum_{n=1}^{m} N_{D_{n}} \cdot I_{D_{n}}}, & \sum_{n=1}^{m} N_{D_{n}} \cdot I_{D_{n}}>H .
\end{array}\right.
$$

We consider the same example as in Section VI. If we assume $a=4$ and $m=10$, then total interference within the effective interference radius $D_{10}$ is $\sum_{n=1}^{10} N_{D_{n}} \cdot I_{D_{n}}=$ 16.5307 and, therefore, the average density of APs per user when the optimal social welfare is achieved is $\langle y\rangle_{\text {opt }}=$ $7 / 16.5307=0.4235$. With $m=30$, on the other hand, $<y>_{\text {opt }}=7 / 16.5653=0.4226$. In addition, total interferences with $m=10$ and $m=30$ are not much different and this justifies the assumption of the effective interference radius $D_{m}$.

\section{REFERENCES}

[1] Y. Benkler, "Overcoming agoraphobia: Building the commons of the digitally networked environment," Harvard Journal of Law \& Technology, vol. 11, no. 2, 1998.

[2] E. Noam, "Spectrum auctions: Yesterday's heresy, today's orthodoxy, tomorrow's anachronism. taking the next step to open spectrum access,' Journal of Law \& Economics, vol. 41, no. S2, pp. 765-790, October 1998.

[3] K. Werbach, "Open spectrum: The new wireless paradigm," new America Foundation, Spectrum Policy Program, Spectrum Series Working Paper \#6 October 2002.

[4] D. P. Reed, "How wireless networks scale: the illusion of spectrum scarcity," in International Symposium On Advanced Radio Technologies (ISART), 2002.

[5] M. H. Manshaei, J. Freudiger, M. Felegyhazi, P. M. adn, and J.-P. Hubaux, "On wireless social community networks," in INFOCOM, 2008. 
[6] G. Hardin, "The tragedy of the commons," Science, vol. 162, pp. 1243 $-1248,1968$.

[7] D. Satapathy and J. Peha, "Etiquette modification for unlicensed spectrum: approach and impact," Vehicular Technology Conference, 1998. VTC 98. 48th IEEE, vol. 1, pp. 272-276, 1998.

[8] W. Lehr and J. Crowcroft, "Managing shared access to a spectrum commons," New Frontiers in Dynamic Spectrum Access Networks, 2005. DySPAN 2005. 2005 First IEEE International Symposium on, pp. 420444, 2005.

[9] D. Monderer and L. S. Shapley, "Potential games," Games and Economic Behavior, vol. 14, no. 1, pp. 124-143, 1996.

[10] F. Xu, L. Zhang, Z. Zhou, and Q. Liang, "Adaptive power control for cooperative uwb network using potential game theory," Wireless Communications and Networking Conference, 2007.WCNC 2007. IEEE, pp. 1620-1624, March 2007.

[11] T. Heikkinen, "A potential game approach to distributed power control and scheduling," Computer Networks, vol. 50, no. 13, pp. 2295-2311, September 2006.

[12] M. Xiao, N. Shroff, and E. Chong, "A utility-based power-control scheme in wireless cellular systems," Networking, IEEE/ACM Transactions on, vol. 11, no. 2, pp. 210-221, April 2003.

[13] E. Altman, Y. Hayel, and H. Kameda, "Evolutionary dynamics and potential games in non-cooperative routing," Modeling and Optimization in Mobile, Ad Hoc and Wireless Networks and Workshops, 2007. WiOpt 2007. 5th International Symposium on, pp. 1-5, April 2007.

[14] S. Shakkottai, E. Altman, and A. Kumar, "Multihoming of users to access points in wlans: A population game perspective," Selected Areas in Communications, IEEE Journal on, vol. 25, no. 6, pp. 1207-1215, August 2007.

[15] V. Srivastava, J. Neel, A. Mackenzie, R. Menon, L. Dasilva, J. Hicks, J. Reed, and R. Gilles, "Using game theory to analyze wireless ad hoc networks," Communications Surveys \& Tutorials, IEEE, vol. 7, no. 4 pp. 46-56, Quarter 2005.

[16] Y. Xiao, X. Shan, and Y. Ren, "Game theory models for ieee $802.11 \mathrm{dcf}$ in wireless ad hoc networks," Communications Magazine, IEEE, vol. 43, no. 3, pp. S22-S26, March 2005.

[17] L. Chen and J. Leneutre, "On the power and rate control in ieee 802.11 wlans - a game theoretical approach," Computer Communications and Networks, 2007. ICCCN 2007. Proceedings of 16th International Conference on, pp. 450-456, Aug. 2007.

[18] J. Sun, E. Modiano, and L. Zheng, "Wireless channel allocation using an auction algorithm," Selected Areas in Communications, IEEE Journal on, vol. 24, no. 5, pp. 1085-1096, 2006.

[19] R. Etkin, A. Parekh, and D. Tse, "Spectrum sharing for unlicensed bands," Selected Areas in Communications, IEEE Journal on, vol. 25, no. 3, pp. 517-528, 2007.

[20] K. Leung and B.-J. Kim, "Frequency assignment for ieee 802.11 wireless networks," Vehicular Technology Conference, 2003. VTC 2003-Fall. 2003 IEEE 58th, vol. 3, pp. 1422-1426, Oct. 2003.

[21] D. Mishra and D. C. Parkes, "Ascending price vickrey auctions for general valuations," August 2005, cORE Discussion Paper No. 2005/52.

[22] P. Mahonen, J. Riihijarvi, and M. Petrova, "Automatic channel allocation for small wireless local area networks using graph colouring algorithm approach," Personal, Indoor and Mobile Radio Communications, 2004. PIMRC 2004. 15th IEEE International Symposium on, vol. 1, pp. 536539, Sept. 2004.

[23] J. Riihijarvi, M. Petrova, and P. Mahonen, "Frequency allocation for wlans using graph colouring techniques," Wireless On-demand Network Systems and Services, 2005. WONS 2005. Second Annual Conference on, pp. 216-222, Jan. 2005.

[24] F. Reif, Fundamentals of Statistical and Thermal Physics. McGrawHill, 1985. 